\title{
Reconfigurable Intelligent Surface-Assisted Uplink Sparse Code Multiple Access
}

\author{
Ibrahim Al-Nahhal, Member, IEEE, Octavia A. Dobre, Fellow, IEEE, and Ertugrul Basar, Senior Member, IEEE
}

\begin{abstract}
Reconfigurable intelligent surface-empowered communication (RIS) and sparse code multiple access (SCMA) are promising candidates for future generations of wireless networks. The former enhances the transmission environments, whereas the latter provides a high spectral efficiency transmission. This letter proposes, for the first time, an RIS-assisted uplink SCMA (SCMA-RIS) scheme to improve the conventional SCMA spectrum efficiency. The message passing algorithm (MPA) is utilized and modified to decode the SCMA-RIS transmitted signals. Moreover, a low-complexity decoder for the SCMA-RIS scheme is proposed to significantly reduce the MPA decoding complexity and improve the bit error rate performance of the conventional SCMA. Monte-Carlo simulations and complexity analysis are presented, which support the findings.
\end{abstract}

Index Terms-Sparse code multiple access (SCMA), reconfigurable intelligent surface (RIS), message passing algorithm (MPA), low-complexity decoder.

\section{INTRODUCTION}

$\mathbf{S}$ PARSE code multiple access (SCMA) is a code-domain non-orthogonal multiple access (C-NOMA) approach; it represents a promising candidate for beyond $5 \mathrm{G}$ wireless networks that can provide a high spectral efficiency transmission [1]-[4]. In the SCMA scheme, a unique multi-carrier sparse code is assigned to each user to share the wireless medium [5], [6]. The message passing algorithm (MPA) can be utilized to decode the SCMA transmitted signals due to the sparsity property of the codes. The MPA is an iterative algorithm that provides a near maximum likelihood (ML) bit error rate (BER) performance with a relatively lower decoding complexity [7].

Reconfigurable intelligent surface (RIS)-empowered communication is another promising technology for beyond 5G wireless networks, which enhances the transmission environment for wireless communication schemes [8], [9]. Without a need for coding or encoding, an RIS adjusts the incident signals' phases using low-cost passive reflecting elements to enhance the signal quality at the receiver side [10]. Recently, RIS and power-domain NOMA have been jointly explored in [11]-[13]. It is worth noting that the power-domain and C-NOMA are completely different medium access schemes, which share the users' data using powers and codes, respectively. To the best of the authors' knowledge, C-NOMA, especially SCMA, has not been investigated yet under the RIS scenario.

For the first time, this letter explores the uplink SCMA scheme assisted by an RIS (SCMA-RIS), to improve the

O. A. Dobre and I. Al-Nahhal are with the Faculty of Engineering and Applied Science, Memorial University, St. John's, NL, Canada, (e-mail: \{odobre, ioalnahhal\}@mun.ca).

E. Basar is with the CoreLab, Department of Electrical and Electronics Engineering, Koç University, Istanbul, Turkey (e-mail: ebasar@ku.edu.tr). spectral efficiency of the conventional SCMA scheme. The conventional ML and MPA decoders are adapted to decode the proposed SCMA-RIS transmitted signals. Furthermore, a novel non-iterative low-complexity (LC) decoder for the SCMARIS, referred to as SCMA-RIS-LC, is proposed to overcome the high decoding complexity of the iterative MPA. Compared to the conventional SCMA-MPA scheme, SCMA-RIS-MPA provides significantly enhanced BER performance with slight increase in the decoding complexity, while the SCMA-RIS-LC shows its superiority in both BER performance and decoding complexity. Monte-Carlo simulations and complexity analysis are provided to evaluate our findings.

The rest of the letter is organized as follows: Sections $\Pi$ and III) introduce the system model and the detection algorithms for the proposed uplink SCMA-RIS, respectively. In Section IV simulation results are presented. Finally, Section $\nabla$ concludes the letter.

\section{UPLINK SCMA-RIS SYSTEM MODEL}

Consider $U$ users which deliver their data to the base station using the SCMA scheme, through an RIS with $N$ reflecting elements, as illustrated in Fig. 1. Each user accesses the medium by employing a unique sparse codebook, $\mathbf{C}_{u} \in \mathbb{C}^{R \times M}$, $u=1, \ldots, U$, which is spread over $R$ orthogonal resource elements (OREs). It is worth noting that each user's codebook contains $M$ codewords, $\mathbf{c}_{u, m} \in \mathbb{C}^{R \times 1}, m=1, \ldots, M$, which have $d_{v}$ non-zero codeword elements. The $U$ users are overloaded over OREs such that the number of users that share each ORE, $d_{f}$, is fixed.

As shown in Fig. 1, each user transmits a codeword, $\mathbf{c}_{u, m}$, which corresponds to $\log _{2}(M)$ bits through $N$ passive reflecting elements. The task of the RIS elements is to adjust the phase of the incident signal to improve the signal quality at the receiver side. The noisy signal at the receiver side for each ORE, $y^{r}$, is

$$
y^{r}=\sum_{u \in \Lambda_{r}}\left(\sum_{n=1}^{N} h_{u, n}^{r} e^{\jmath \phi_{n}^{r}} g_{n}^{r}\right) c_{u, m}^{r}+w^{r},
$$

where $h_{u, n}^{r}$ and $g_{n}^{r}$ represent the Rayleigh fading channel coefficient for the $r$-th ORE from the $u$-th user to the $n$-th reflecting element and from the $n$-th reflecting element to the receiver, respectively. Here, $\Lambda_{r}$ represents the set of users' indices that share the $r$-th ORE, $\phi_{n}^{r}$ denotes the adjustable phase produced by the $n$-th reflecting element at the $r$-th ORE, and $w^{r} \sim \mathcal{N}\left(0, \sigma^{2}\right)$ is the additive white Gaussian noise at the $r$-th ORE with zero-mean and variance of $\sigma^{2}$. It is worth noting that since the OREs for the SCMA can 
Fig. 1. Uplink SCMA-RIS scheme.

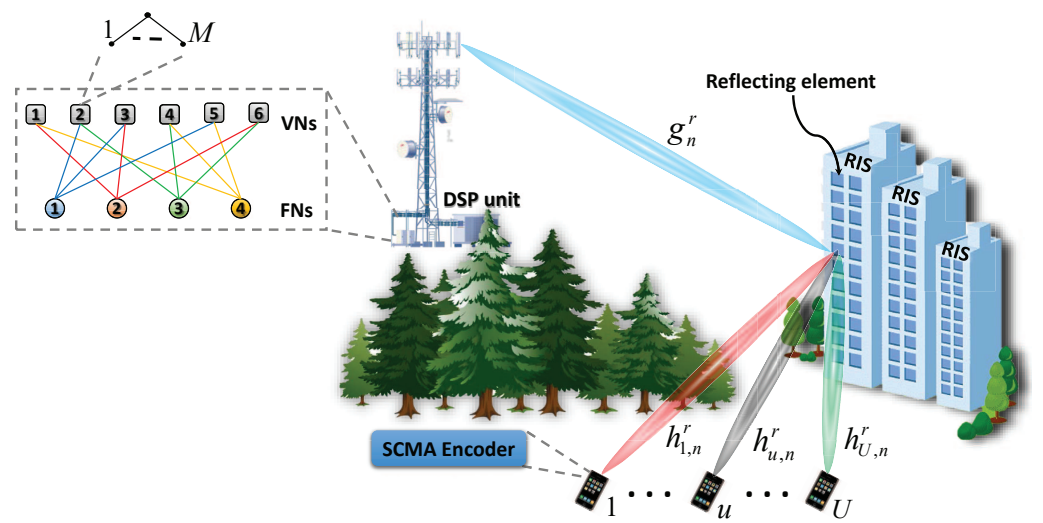

be orthogonal time or frequency elements, the assumption of having an adjustable phase for each ORE is feasible.

The channel coefficients, $h_{u, n}^{r}$ and $g_{u}^{r}$, can be written in the polar form, respectively as

$$
h_{u, n}^{r}=\alpha_{u, n}^{r} e^{-\jmath\left(\theta_{u, n}^{r}\right)}, \quad g_{n}^{r}=\beta_{n}^{r} e^{-\jmath\left(\vartheta_{n}^{r}\right)},
$$

Hence, by plugging (2) into (1), the received signal, $y^{r}$, can be expressed as

$$
y^{r}=\sum_{u \in \Lambda_{r}}\left(\sum_{n=1}^{N} \alpha_{u, n}^{r} \beta_{n}^{r} e^{\jmath\left(\Theta_{u, n}^{r}\right)}\right) c_{u, m}^{r}+w^{r},
$$

where $\Theta_{u, n}^{r}=\phi_{n}^{r}-\left(\theta_{u, n}^{r}+\vartheta_{n}^{r}\right)$ represents the resulted phase after the adjustment of the $n$-th reflecting RIS element for the $u$-th user at the $r$-th ORE. To improve the instantaneous signal-to-noise ratio (SNR) at the $r$-th ORE in (3), $\phi_{n}^{r}$ can be obtained as

$$
\phi_{n}^{r}=\underset{\phi_{n}^{r} \in[-\pi, \pi]}{\arg \min } \sum_{u \in \Lambda_{r}}\left|\phi_{n}^{r}-\left(\theta_{u, n}^{r}+\vartheta_{n}^{r}\right)\right|^{2} .
$$

The optimum solution for (4) is the geometric median of the users' phases, which share that ORE, i.e., $\phi_{n}^{r}=\operatorname{med}\left(\theta_{u, n}^{r}+\right.$ $\vartheta_{n}^{r}$ ) for all $u \in \Lambda_{r}$, with $\operatorname{med}($.$) denoting the geometric$ median. Consequently, the values of $\theta_{u, n}^{r}$ and $\vartheta_{n}^{r}$ should be known at RIS to optimize $\phi_{n}^{r}$. To notice the improvement in the BER performance after optimizing $\phi_{n}^{r}$, we consider a blind scenario in which the reflector elements of RIS are reflecting the incident signals without any adjustment to the phases (i.e., $\phi_{n}^{r}=0$ for $\forall n$ ). The two scenarios of the $\phi_{n}^{r}$ can be summarized as

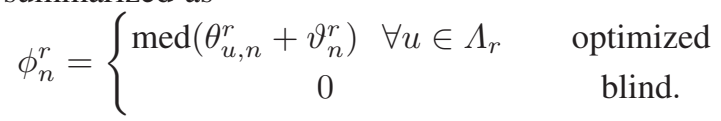

The vector of received signals, $\mathbf{y}$, for the SCMA-RIS scheme for all OREs is

$$
\mathbf{y}=\sum_{u=1}^{U}\left(\operatorname{diag}\left(\boldsymbol{H}_{u}\right) \mathbf{c}_{u, m}\right)+\mathbf{w}
$$

where $\mathbf{y} \in \mathbb{C}^{R \times 1}=\left[y^{1}, \ldots, y^{R}\right]^{\mathrm{T}}, \mathbf{w} \in \mathbb{C}^{R \times 1}=$ $\left[w^{1}, \ldots, w^{R}\right]^{\mathrm{T}}$, and $\boldsymbol{H}_{u} \in \mathbb{C}^{R \times 1}$ is

$$
\boldsymbol{H}_{u}=\left[\left(\sum_{n=1}^{N} \alpha_{u, n}^{1} \beta_{n}^{1} e^{\jmath\left(\Theta_{u, n}^{1}\right)}\right), \ldots,\left(\sum_{n=1}^{N} \alpha_{u, n}^{R} \beta_{n}^{R} e^{\jmath\left(\Theta_{u, n}^{R}\right)}\right)\right]_{(7)}^{\mathrm{T}},
$$

where $\operatorname{diag}\left(\boldsymbol{H}_{u}\right) \in \mathbb{C}^{R \times R}$ represents a diagonal matrix whose $r$-th diagonal element is $\sum_{n=1}^{N} \alpha_{u, n}^{r} \beta_{n}^{r} e^{\jmath\left(\Theta_{u, n}^{r}\right)}$.

\section{UPLINK SCMA-RIS SIGNAL DETECTION}

In this section, we presents three decoders for the proposed uplink SCMA-RIS scheme, which are ML, MPA and LC decoders. The complexity analysis is also provided.

\section{A. SCMA-RIS-ML Decoder}

In SCMA-RIS, the ML decoder provides theoretical the optimum BER performance by performing an exhaustive search for all $(M)^{U}$ users' codeword combinations. The estimated transmitted codewords for all users, $\hat{\mathbf{C}}_{\mathrm{ML}} \in \mathbb{C}^{R \times U}=$ $\left[\hat{\mathbf{c}}_{1, m} \ldots \hat{\mathbf{c}}_{U, m}\right]$, using the ML decoder can be expressed as

$$
\hat{\mathbf{C}}_{\mathrm{ML}}=\underset{j \in M^{U}}{\arg \min }\left\|\mathbf{y}-\sum_{u=1}^{U}\left(\operatorname{diag}\left(\boldsymbol{H}_{u}\right) \mathbf{c}_{u, m(j)}\right)\right\|^{2}
$$

where $m(j)$ is the value of $m \in\{1, \ldots, M\}$ at the $(M)^{U}$ codeword combination. However, the SCMA-RIS-ML is not used due to its impractical implementation.

\section{B. SCMA-RIS-MPA Decoder}

The MPA is an iterative decoder that provides a near ML BER performance at an implementable decoding complexity for the SCMA-RIS scheme. The SCMA-RIS-MPA uses a factor graph method as depicted in Fig. 11 The MPA iteratively updates the probability of the messages between the function nodes (FNs) representing the OREs and variable nodes (VNs) that represent the served users. The MPA stops after $K$ iterations to detect the users' codewords that correspond to the maximum joint message probability. It is worth noting that the conventional SCMA-MPA is modified to estimate the SCMA-RIS transmitted codewords.

To formulate the MPA, consider that $\mathcal{P}_{v_{u} \rightarrow f_{r}}^{(t)}\left(c_{m}^{r, u}\right)$ and $\mathcal{P}_{f_{r} \rightarrow v_{u}}^{(t)}\left(c_{m}^{r, u}\right)$ represent the probability of passing the message from the $u$-th $\mathrm{VN}$ to the $r$-th $\mathrm{FN}$ and from the $r$-th $\mathrm{FN}$ to the $u$-th $\mathrm{VN}$, respectively, at the $k$-th iteration, $k=1, \ldots, K$. At the first iteration, all messages sent from VNs to FNs are assumed equiprobable; i.e., 


$$
\mathcal{P}_{v_{u} \rightarrow f_{r}}^{(0)}\left(c_{m}^{r, u}\right)=\frac{1}{M}, \quad \forall u, \forall r, \forall m .
$$

Thus, $\mathcal{P}_{f_{r} \rightarrow v_{u}}^{(k+1)}\left(c_{m}^{r, u}\right)$ can be written as

$$
\begin{aligned}
& \mathcal{P}_{f_{r} \rightarrow v_{u}}^{(k+1)}\left(c_{m}^{r, u}\right)=\sum_{\psi(i), i \in \Lambda_{r} \backslash u}\left\{\mathcal{P}\left(\mathbf{y} \mid \psi(i), \psi(u)=c_{m}^{r, u}\right)\right. \\
& \left.\quad \times \prod_{i \in \Lambda_{r} \backslash u} \mathcal{P}_{v_{i} \rightarrow f_{r}}^{(k)}(\psi(i))\right\}, \quad \forall m, \forall r, u \in \Lambda_{r},
\end{aligned}
$$

where $\Lambda_{r} \backslash u$ represents $\Lambda_{r}$ except the $u$-th user, $\boldsymbol{\psi}^{r}=$ $\{\psi(1), \ldots, \psi(i), \ldots\}$ denotes the possible codewords of all users that share the $r$-th ORE, and

$$
\begin{aligned}
& \mathcal{P}\left(\mathbf{y} \mid \boldsymbol{\psi}^{r}\right)=\frac{1}{\sqrt{2 \pi} \sigma} \\
& \times \exp \left(-\frac{\left|y^{r}-\sum_{u \in \Lambda_{r}}\left(\left(\sum_{n=1}^{N} \alpha_{u, n}^{r} \beta_{n}^{r} e^{\jmath\left(\Theta_{u, n}^{r}\right)}\right) c_{m}^{r, u}\right)\right|^{2}}{2 \sigma^{2}}\right) .
\end{aligned}
$$

It should be noted that the effect of RIS obviously appears in (11). Now, $\mathcal{P}_{v_{u} \rightarrow f_{r}}^{(k+1)}\left(c_{m}^{r, u}\right)$ can be updated as

$$
\begin{aligned}
& \mathcal{P}_{v_{u} \rightarrow f_{r}}^{(k+1)}\left(c_{m}^{r, u}\right)=\gamma_{u, r}^{(k+1)} \\
& \quad \times \prod_{j \in \Omega_{u} \backslash r} \mathcal{P}_{f_{r} \rightarrow v_{u}}^{(k+1)}\left(c_{m}^{r, u}\right), \quad \forall m, \forall u, r \in \Omega_{u},
\end{aligned}
$$

where $\Omega_{u}$ represents the OREs' indices that correspond to $d_{v}$ non-zero positions of the $u$-th user, $\Omega_{u} \backslash r$ denotes $\Omega_{u}$ except the $r$-th ORE, and $\gamma_{u, r}^{(k+1)}$ is

$$
\gamma_{u, r}^{(k+1)}=\left(\sum_{m=1}^{M} \mathcal{P}_{v_{u} \rightarrow f_{r}}^{(k)}\left(c_{m}^{r, u}\right)\right)^{-1}
$$

After $K$ iterations, the estimated transmitted codeword of the $u$-th user can be given by

$$
\begin{aligned}
\left\{\hat{\mathbf{c}}_{m}^{u}\right\}^{(K)} & =\underset{m}{\arg \max } \prod_{j \in \Omega_{u}} \mathcal{P}_{f_{j} \rightarrow v_{u}}^{(K)}\left(c_{m}^{r, u}\right), \forall u . \\
m, \ldots, M & .
\end{aligned}
$$

The set of all estimated transmitted users' codewords using the SCMA-RIS-MPA decoder, $\hat{\mathbf{C}}_{\mathrm{MPA}}$, is

$$
\hat{\mathbf{C}}_{\mathrm{MPA}}=\left\{\left\{\hat{\mathbf{c}}_{m}^{1}\right\}^{(K)}, \ldots,\left\{\hat{\mathbf{c}}_{m}^{U},\right\}^{(K)}\right\} .
$$

\section{SCMA-RIS-LC Decoder}

In this subsection, an LC algorithm for the SCMA-RIS scheme is proposed and analyzed. The SCMA-RIS-LC is a non-iterative decoder that significantly reduces the decoding complexity to overcome the high complexity of the MPA. The proposed SCMA-RIS-LC decoder performs two stages to decode the transmitted users' codewords, as follows:

Stage 1: In this stage, the SCMA-RIS-LC decoder detects the transmitted users' codewords using a single ORE by trying all possible combinations between the users' codewords, which share that ORE. The detected users' codewords are employed to sequentially detect the rest of the users' codewords for the other OREs. This stage stops when all users' codewords are detected. It should be noted that the detected users' codewords from this stage represent the initial values for the next stage. The detected users' codewords from Stage $1, \hat{\mathbf{C}}_{\mathrm{LC}}$, is

$$
\begin{aligned}
\hat{\mathbf{C}}_{\mathrm{LC}} & =\underset{j}{ }=1, \ldots, M^{\grave{U}^{r}} \\
& \arg \min \\
& =1, \Psi_{1}-\left.\Psi_{2}(j)\right|^{2}, \quad 1 \leq r \leq R,
\end{aligned}
$$

where $\Psi_{1}$ and $\Psi_{2}$ respectively represent the users' signals that have already been detected and that need to be detected at the $r$-th ORE, given as

$$
\begin{gathered}
\Psi_{1}=\sum_{u \in \grave{\Lambda}_{r}}\left(\sum_{n=1}^{N} \alpha_{u, n}^{r} \beta_{n}^{r} e^{\jmath\left(\Theta_{u, n}^{r}\right)}\right) \hat{c}_{u, m}^{r} \mid \hat{c}_{u, m}^{r} \subset \hat{\mathbf{C}}_{\mathbf{L C}}, \\
\Psi_{2}(j)=\sum_{u \in \Lambda_{r} \backslash \grave{\Lambda}_{r}}\left(\sum_{n=1}^{N} \alpha_{u, n}^{r} \beta_{n}^{r} e^{\jmath\left(\Theta_{u, n}^{r}\right)}\right) c_{u, m(j)}^{r} .
\end{gathered}
$$

Here, $\grave{\Lambda}_{r}$ denotes the users' indices that share the $r$-th ORE and their codewords are already detected, $\Lambda_{r} \backslash \grave{\Lambda}_{r}$ represents $\Lambda_{r}$ except $\grave{\Lambda}_{r}$ (i.e., the user's indices whose codewords need to be detected), and $\grave{U}^{r}=\operatorname{card}\left\{\Lambda_{r} \backslash \grave{\Lambda}_{r}\right\} \leq d_{f}$ with $\operatorname{card}\{\cdot\}$ as the number of set elements.

Stage 2: In this stage, a single user's codeword is detected at a time using his $d_{v}$ non-zero OREs that carry its codeword. Also, all other users' codewords are considered to be known from Stage 1 (or from Stage 2 if they have been detected already). The detected user's codeword, $\hat{\mathbf{c}}_{m}^{u}$, is

$$
\hat{\mathbf{c}}_{m}^{u}=\underset{j=1, \ldots, M}{\arg \min } \sum_{r \in \Omega_{u}}\left|y^{r}-\Psi_{3}-\Psi_{4}(j)\right|^{2}, u=1, \ldots, U,
$$

where

$$
\begin{gathered}
\Psi_{3}=\sum_{\grave{u} \in \Lambda_{r} \backslash u}\left(\sum_{n=1}^{N} \alpha_{\grave{u}, n}^{r} \beta_{n}^{r} e^{\jmath\left(\Theta_{\grave{u}, n}^{r}\right)}\right) \hat{c}_{\grave{u}, m}^{r} \mid \hat{c}_{\grave{u}, m}^{r} \subset \hat{\mathbf{C}}_{\mathrm{LC}}, \\
\Psi_{4}(j)=\left(\sum_{n=1}^{N} \alpha_{u, n}^{r} \beta_{n}^{r} e^{\jmath\left(\Theta_{u, n}^{r}\right)}\right) c_{u, m(j)}^{r} .
\end{gathered}
$$

It is worth noting that $\Psi_{4}$ represents the desired user's information that needs to be detected. Algorithm 1 summarizes the procedure of the SCMA-RIS-LC decoder.

\section{Complexity Analysis}

In this subsection, the decoding complexity of the proposed SCMA-RIS decoders is deduced in terms of the real additions (RA) and real multiplications (RM) that are required to decode the users' codewords. Table $\Pi$ presents the complexity summary for the two proposed decoders of the SCMA-RIS scheme and the conventional SCMA-MPA. It is worth noting that the summation term in the RA and RM expressions for the SCMA-RIS-LC decoder depends on the system parameters; for $U=6, R=4$ with $d_{f}=3$ it becomes $M^{3}+M^{2}+M$. Numerical comparisons will be given next. 
TABLE I

THE DECODING COMPLEXITY OF THE SCMA-RIS DECODERS.

\begin{tabular}{|c|c|c|}
\hline & Real Additions (RA) & Real Multiplications (RM) \\
\hline SCMA-MPA & 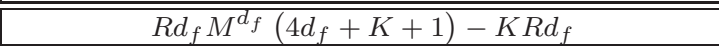 & $\bar{R} R d_{f} M^{d_{f}}\left(4 d_{f}+K d_{f}+3\right)+M\left(d_{v}-1\right)\left(K R d_{f}+U\right)$ \\
\hline SCMA-RIS-MPA & $R d_{f} M^{d_{f}}\left(4 d_{f}+K+1\right)+R d_{f}(N-K)+1$ & $R d_{f} M^{d_{f}}\left(4 d_{f}+K d_{f}+3\right)+M\left(d_{v}-1\right)\left(K R d_{f}+U\right)+R d_{f} N$ \\
\hline SCMA-RIS-LC & $R\left(2 d_{f}-1\right)+\left(4 d_{f}+1\right)\left(\begin{array}{c}U M+\sum_{r}^{R}=1^{M^{U^{r}}} \\
\dot{U}^{r} \neq 0\end{array}\right.$ & $2 R d_{f}+\left(4 d_{f}+2\right)\left(U M+\sum_{\substack{r=1 \\
\dot{U}^{r} \neq 0}}^{R} M^{\dot{U}^{r}}\right)+R d_{f} N$ \\
\hline
\end{tabular}

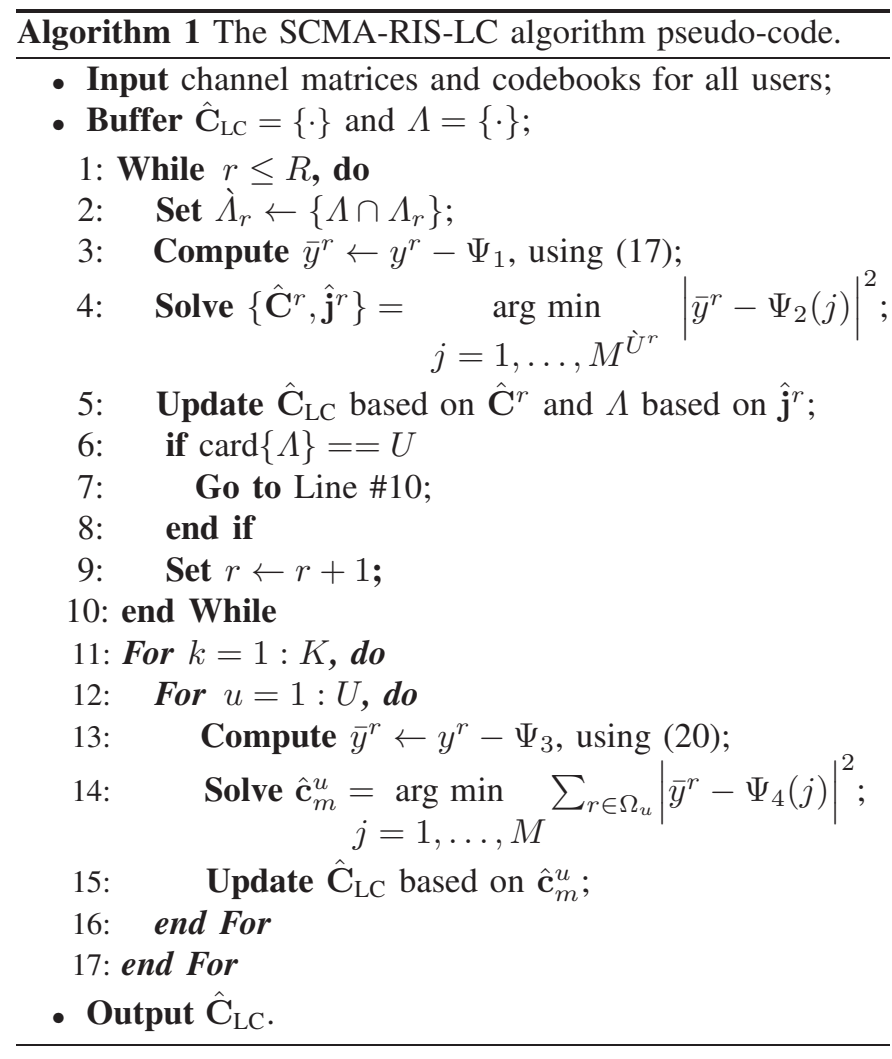

\section{Simulation Results}

In this section, Monte-Carlo simulations are used to assess the BER performance of the proposed SCMA-RIS schemes compared to the conventional SCMA-MPA scheme. It is assumed that the Rayleigh fading channels of all users are perfectly known at the receiver. In our setup, we consider $U=6, R=4$ with $d_{f}=3, M=4$ and 2 , and $N=20,30$ and 40. Moreover, the assessment of the blind and optimized scenarios in (5) for the proposed SCMA-RIS schemes is presented.

The number of iterations, $K$, used in MPA for the proposed SCMA-RIS-MPA and conventional SCMA-MPA is 4 . It is worth noting that the improvement of the BER performance for the MPA decoder is saturated at $K=4$ (i.e., no improvement occurs when $K>4$ ) for both SCMA-MPA and SCMA-RISMPA.

Figs. 2 and 3 depict the BER performance comparison between the proposed SCMA-RIS-MPA and conventional SCMA-MPA for $M=2$ and 4 , respectively. As seen from these figures, the two SCMA-RIS-MPA scenarios (i.e., blind and optimized) significantly improve the BER performance compared to the conventional SCMA-MPA. For instance, the blind SCMA-RIS-MPA scheme for $M=2$ and 4 provides around $13 \mathrm{~dB}, 15 \mathrm{~dB}$ and $16.5 \mathrm{~dB}$ improvement in the BER performance for $N=20,30$ and 40 with $K=4$, respectively, compared to the SCMA-MPA with $K=4$. For the optimized SCMA-RIS-MPA scenario of $M=2$ and 4 , the improvement in the BER performance increases to $22 \mathrm{~dB}, 27 \mathrm{~dB}$ and $31 \mathrm{~dB}$ for $N=20,30$ and 40 with $K=4$, respectively, compared to the SCMA-MPA with $K=4$. Thus, the optimized scenario and an increased number of reflecting elements provide more BER improvements.

On the other hand, the decoding complexity of the SCMARIS-MPA is slightly higher than for the conventional SCMAMPA. For example, from Table \ for $M=2$ and $K=4$, the increase in the number of RA and RM of the SCMARIS-MPA does not exceed $30.4 \%$ and $17.8 \%$ than that of the conventional SCMA-MPA, respectively. Furthermore, when $M=4$, the increase in both RA and RM number for the SCMA-RIS-MPA does not exceed $3.4 \%$.

As seen from Figs. 4, 5 and Table I, the proposed SCMARIS-LC decoder significantly reduces the decoding complexity with a better BER performance than the SCMA-MPA, especially in low SNR. For instance, the reduction in the RA for the SCMA-RIS-LC with $M=2$ is $47 \%, 54.6 \%$ and $62.2 \%$, while it saves around $68 \%, 72.3 \%$ and $76.7 \%$ RM for $N=40,30$ and 20, respectively, compared to SCMA-MPA with $K=4$. For $M=4$, the saving in the complexity of the proposed SCMA-RIS-LC decoder enhances compared to the SCMAMPA with $K=4$; it is around $85.4 \%, 86.3 \%$ and $87.2 \%$ in the RA, whereas it reaches $90.4 \%, 91 \%$ and $91.5 \%$ for RM when $N=40,30$ and 20, respectively. Thus, as $N$ decreases, the saving in the complexity increases, whereas the improvement of the BER performance decreases.

Finally, the proposed SCMA-RIS-MPA provides a significant improvement of the BER performance at the expense of an acceptable increase in the complexity when compared with the conventional SCMA-MPA. Further, the proposed SCMA-RIS-LC significantly reduces the complexity with a considerable improvement in the BER performance when compared with SCMA-MPA.

\section{CONClusion}

In this letter, we have investigated the SCMA under two RIS scenarios, referred to as blind and optimized SCMA-RIS. The MPA is adapted to decode the SCMA-RIS transmitted signals. Furthermore, a low-complexity decoder is proposed and analyzed for the SCMA-RIS, i.e., SCMA-RIS-LC. It is shown that the SCMA-RIS-MPA provides up to $31 \mathrm{~dB}$ improvement in the BER performance with an increase in the 


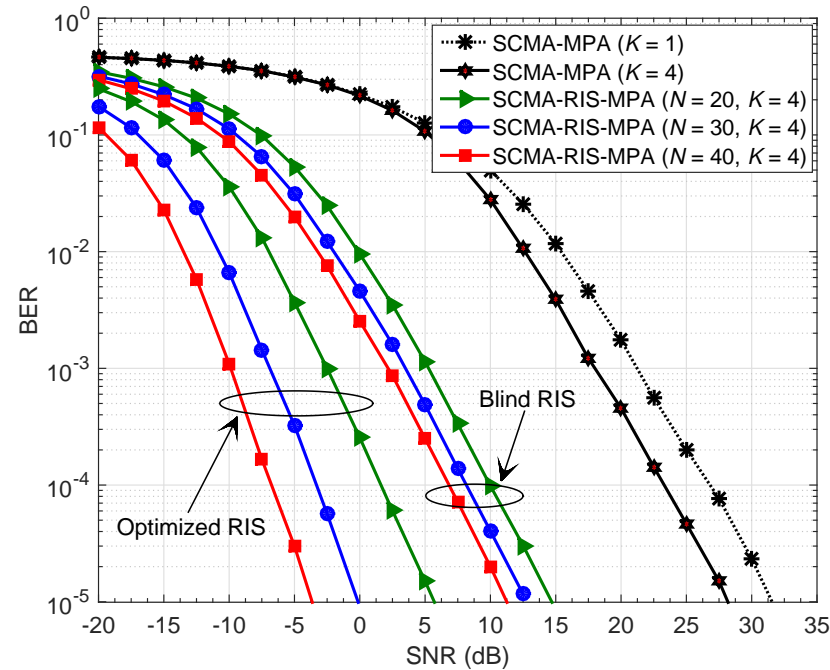

Fig. 2. BER performance comparison of the SCMA-MPA and SCMARIS-MPA schemes for $M=2$.

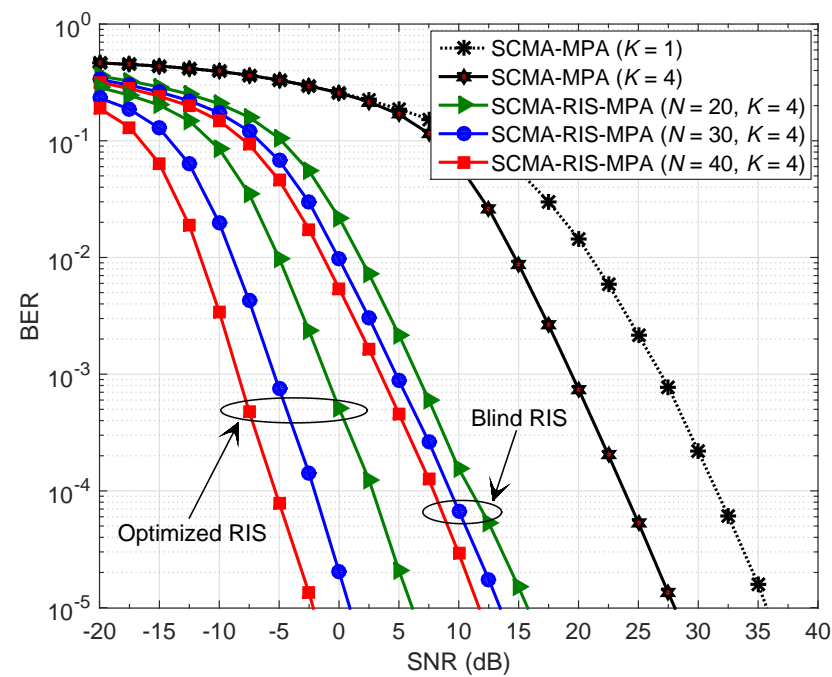

Fig. 3. BER performance comparison of the SCMA-MPA and SCMARIS-MPA schemes for $M=4$

decoding complexity that varies from $30.4 \%$ to less than $3.4 \%$, compared to the SCMA-MPA. Furthermore, the proposed SCMA-RIS-LC reduces the decoding complexity in a range of $47 \%$ to $91.5 \%$ with a better BER performance, compared to the SCMA-MPA.

\section{REFERENCES}

[1] M. Mohammadkarimi, M. A. Raza, and O. A. Dobre, "Signature-based nonorthogonal massive multiple access for future wireless networks: Uplink massive connectivity for machine-type communications," IEEE Veh. Technol. Mag., vol. 13, no. 4, pp. 40-50, Dec. 2018.

[2] S. M. R. Islam et al., "Power-domain non-orthogonal multiple access (NOMA) in 5G systems: Potentials and challenges," IEEE Commun. Surv. Tuts., vol. 19 , no. 2, pp. 721-742, Oct. 2016 .

[3] Z. Ding et al., "A survey on non-orthogonal multiple access for 5G networks: Research challenges and future trends," IEEE J. Sel. Areas Commun., vol. 35, no. 10, pp. 2181-2195, Oct. 2017.

[4] I. Al-Nahhal, O. A. Dobre, E. Basar, and S. Ikki, "Low-cost uplink sparse code multiple access for spatial modulation," IEEE Trans. Veh. Technol., vol. 68, no. 9, pp. 9313-9317, Jul. 2019.

[5] H. Nikopour and H. Baligh, "Sparse code multiple access," in Proc. IEEE Int. Symposium on Personal Indoor and Mobile Radio Commun. (PIMRC), Sep. 2013, pp. 332-336.

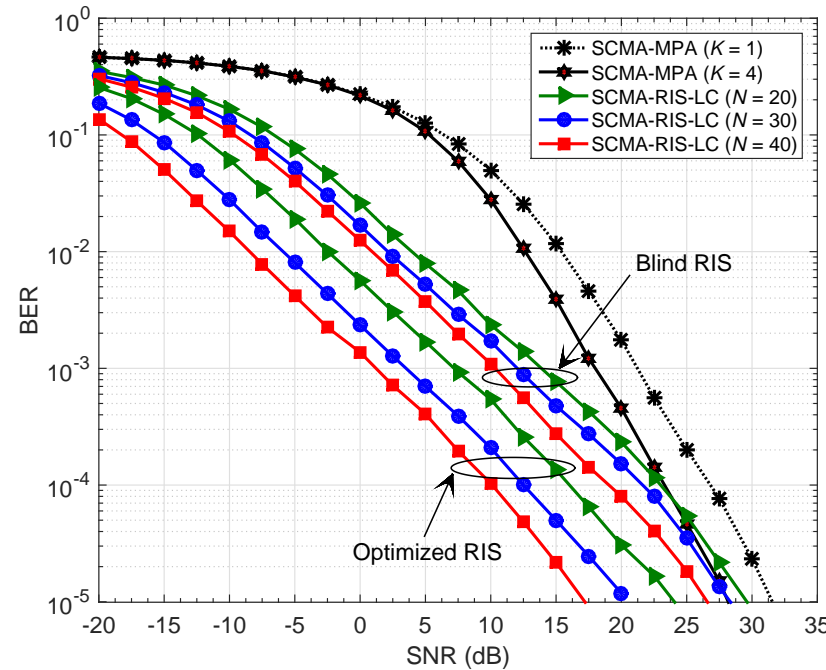

Fig. 4. BER performance comparison of the SCMA-MPA and SCMARIS-LC schemes for $M=2$.

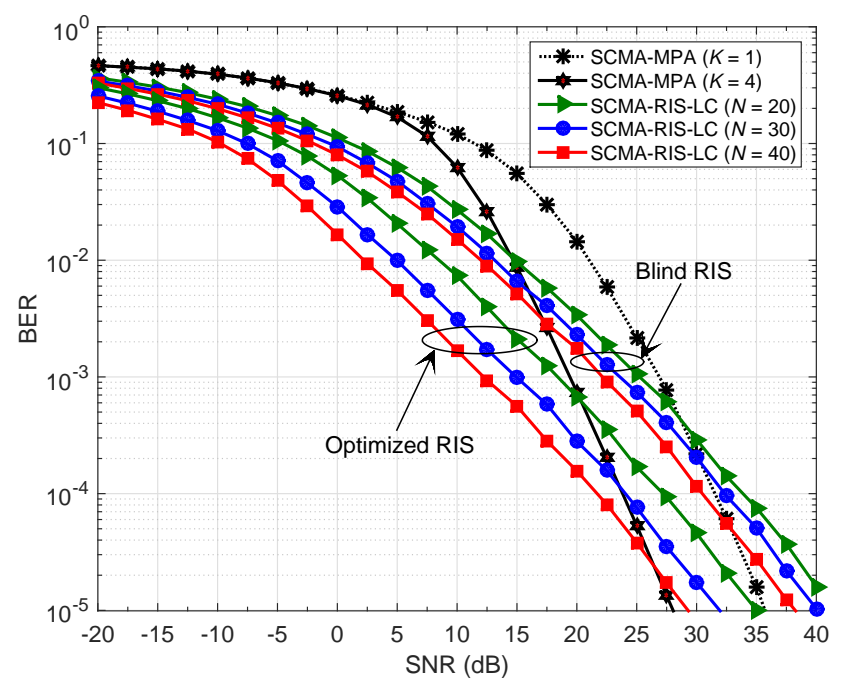

Fig. 5. BER performance comparison of the SCMA-MPA and SCMARIS-LC schemes for $M=4$.

[6] M. Taherzadeh et al., "SCMA codebook design," in Proc. IEEE Veh. Technol. Conf. (VTC Fall), Sep. 2014, pp. 1-5.

[7] H. Mu, Z. Ma, M. Alhaji, P. Fan, and D. Chen, "A fixed low complexity message pass algorithm detector for up-link SCMA system," IEEE Wireless Commun. Lett., vol. 4, no. 6, pp. 585-588, Dec. 2015.

[8] E. Basar, M. D. Renzo, J. de Rosny, M. Debbah, M.-S. Alouini, and R. Zhang, "Wireless communications through reconfigurable intelligent surfaces," IEEE Access, vol. 7, pp. 116753-116773, Sep. 2019.

[9] M. A. ElMossallamy et al., "Reconfigurable intelligent surfaces for wireless communications: Principles, challenges, and opportunities," IEEE Trans. Cogn. Commun. and Netw., vol. 6, pp. 990-1002, Sep. 2020.

[10] E. Basar, "Transmission through large intelligent surfaces: A new frontier in wireless communications," in Proc. European Conf. Netw. Commun. (EuCNC), Aug. 2019, pp. 112-117.

[11] T. Hou, Y. Liu, Z. Song, X. Sun, Y. Chen and L. Hanzo, "Reconfigurable intelligent surface aided NOMA networks," IEEE J. Sel. Areas Commun., Early Access, 2020, doi: 10.1109/JSAC.2020.3007039.

[12] X. Liu et al., "RIS enhanced massive non-orthogonal multiple access networks: Deployment and passive beamforming design," IEEE J. Sel. Areas Commun., Early Access, 2020, doi: 10.1109/JSAC.2020.3018823.

[13] M. Elhattab et al., "Reconfigurable intelligent surface assisted coordinated multipoint in downlink NOMA networks," IEEE Commun. Lett., Early Access, 2020, doi: 10.1109/LCOMM.2020.3029717. 